Recepción: 20 / 12 / 2017

Aceptación: 15 / 02 / 2018

Publicación: 8 / 05 / 2018
Ciencia Económica

Artículo de Revisión

\title{
Auditoría de Gestión como técnica de análisis de las Operaciones Empresariales
}

\author{
Management Audit as a technique for \\ analyzing Business Operations \\ Auditoria de Gestão como técnica de \\ análise para Operações de Negócios
}

Narda P. Merchán-Ponce ${ }^{I}$

nardapmp@hotmail.com

Nancy F. Pinargote-Vásquez II

n_pinargote@yahoo.com

Erick R. Baque-Sanchez III

erickbaque@hotmail.com

\author{
Gloria P. Chiquito-Tigua IV \\ g_chiquito@hotmail.com \\ Ruben D. Acebo-Calderón V \\ rubendac1@yahoo.es \\ Lady L. Paredes-Acebo VI \\ laura.pardesacebo@gmail.com
}

Correspondencia: nardapmp@ hotmail.com

I. Magister en Administración de Empresas mención Dirección Financiera, Universidad Estatal del Sur de Manabí, Manta Ecuador.

II. Magister en Auditoria de Sistemas de Gestión de la Calidad, Universidad Laica Eloy Alfaro de Manabí, Manta Ecuador.

III. Magister en Contabilidad y Auditoría, Universidad Estatal del Sur de Manabí, Manta Ecuador.

IV. Magister en Contabilidad y Auditoría, Universidad Estatal del Sur de Manabí, Manta Ecuador.

V. Diplomado en Evaluación y Acreditación Universitaria, Universidad Estatal del Sur de Manabí, Manta Ecuador.

VI. Estudiante del Sexto Semestre, Carrera de Contabilidad y Auditoría, Universidad Tecnológica Indoamérica, Sabanilla, Quito. 
Narda P. Merchán-Ponce; Nancy F. Pinargote-Vásquez; Erick R. Baque-Sanchez; Gloria P. Chiquito-Tigua; Ruben D. Acebo-

\section{Resumen}

La presente investigación se efectuó con la finalidad de describir como técnica de análisis de las operaciones empresariales la auditoría de gestión. Se establecieron los pasos fundamentales para su comprensión; desde la formulación de conceptos básicos, objetivos primordiales, las etapas del marco metodológico y la utilización de indicadores de gestión. La revisión de la técnica señala que es una la herramienta completa para el análisis de la gestión empresarial. La misma permite hacer un examen de la estructura organizativa y de sus sistemas de gestión y control. Por lo tanto se recomienda que a futuros estudios puedan ser plasmados a casos específicos para verificar sus objetivos empresariales con la realidad de la gestión. Entre algunas de las conclusiones se puede afirmar que la auditoria es una herramienta que permite ver completamente las interacciones de las empresas u organizaciones para el análisis de las operaciones empresariales y como recomendación, las empresas que requieran mejorar su gestión, pueden aplicar la auditoría como estrategia de competitividad en el mercado.

Palabras clave: Auditoría, gestión, análisis, operaciones. 


\begin{abstract}
The present investigation was carried out with the purpose of describing the management audit as business analysis technique. The fundamental steps for its understanding were established; from the formulation of basic concepts, primary objectives, the stages of the methodological framework and the use of management indicators. The review of the technique indicates that it is a complete tool for the analysis of business management. It allows an examination of the organizational structure and its management and control systems. Therefore, it is recommended that future studies can be tailored to specific cases to verify their business objectives with the reality of management. Among some of the conclusions we can say that the audit is a tool that allows to see completely the interactions of companies or organizations for the analysis of business operations and as a recommendation, companies that need to improve their management, can apply the audit as a strategy of competitiveness in the market.
\end{abstract}

Keywords: Audit, management, analysis, operations. 
Narda P. Merchán-Ponce; Nancy F. Pinargote-Vásquez; Erick R. Baque-Sanchez; Gloria P. Chiquito-Tigua; Ruben D. Acebo-

\section{Introducción.}

La complejidad empresarial insta a las empresas a mejorar cada día su operatividad para mantenerse vigente en el mercado; la eficacia y la eficiencia se han convertido en pilares fundamentales en la gestión para que la organización pueda generar mejora continua, optimizar los recursos y tomar decisiones a tiempo.

En su estudio, Redondo et al. (1996) definen a la auditoría de gestión como:

Una técnica relativamente nueva de asesoramiento que ayuda a analizar, diagnosticar y establecer recomendaciones a la empresa con el fin de conseguir con éxito una estrategia. Uno de los motivos principales por la cual la empresa puede decir emprender una auditoría de gestión es el cambio que se hace indispensable para reajustar la gestión o la organización de la misma. (p. 2).

Stoner, Freeman, R, \& Gilbert. (1996) respecto al concepto de auditoria también hace mención con lo siguiente

Las auditorías internas sirven a los directivos para evaluar la eficiencia en las operaciones de la organización y el desempeño de los sistemas de control. Este proceso se concentra en las operaciones de la organización, también se conoce como auditoria de las operaciones. (p. 629)

Es imprescindible contar con una herramienta que nos permita identificar cuáles son los ajustes que debemos hacer y cuales estrategias están siendo bien diseñadas y ejecutadas. Según Franklin. (2007) "la auditoria administrativa es la revisión total o parcial de una organización con el propósito 
de precisar su nivel de desempeño y perfilar oportunidades de mejorar para innovar valor y generar una ventaja competitiva sustentable" (p. 11)

De igual manera en su estudio Cubero. (2009) expone que "en general la gestión comprende todas las actividades de una organización que implica el establecimiento de metas y objetivos, así como la evaluación de su desempeño y cumplimiento; además del desarrollo de una estrategia operativa que garantice la supervivencia" (p.24).

La auditoría de gestión como su mismo nombre lo indica se refiere a la gestión, a la operacionalidad de la empresa u organización, en relación a esto, Redondo et al (1996) establecen que la auditoría de gestión examina las practicas de gestión; no existen principios de gestión generalmente aceptados, que estén codificados, sino que se basan en la práctica normal de la gestión; los criterios de evaluación de la gestión han de diseñarse para cada caso en especifico, pudiéndose extender a casos similares; las recomendaciones sobre la gestión han de ser extensas y adaptadas al hecho fiscalizado, analizando las causas de las ineficiencias y sus consecuencias. (p. 2)

Sobre el tema del enfoque de la auditoria operacional Bernal. (2009) argumenta que

El enfoque inicial de una auditoría operacional está dirigido hacia hallar las operaciones o actividades inefectivas, antieconómicas o ineficientes. Encontrarlas constituye el Hallazgo de Auditoría Operacional que se puede definir como la operación o actividad que está afectada negativamente o lesionada en la eficacia, economía o eficiencia. (p. 2).

Esto nos lleva a considerar tres aspectos importantes del control de gestión, como son la Eficacia, la eficiencia y la economía. Según Redondo et al (1996) 
Narda P. Merchán-Ponce; Nancy F. Pinargote-Vásquez; Erick R. Baque-Sanchez; Gloria P. Chiquito-Tigua; Ruben D. Acebo-

La eficacia de una empresa se mide por el grado de cumplimientos de los objetivos contenidos en los programas de actuación de la misma. La eficiencia se mide por los bienes adquiridos o producidos o servicios prestados por un lado (outputs) y los recursos utilizados por el otro (inputs). Y la economía mide las condiciones en que una empresa adquiere los recursos financieros, humanos y materiales. (p. 4)

En su investigación Valencia (2013), expone

El objetivo primordial de la auditoría de gestión consiste en descubrir deficiencias o irregularidades en algunas partes de la empresa y apuntar a sus probables remedios para ayudar a la dirección a lograr la administración más eficaz. Y como objetivos específicos:

- Determinar lo adecuado de la organización de la entidad.

- Verificar la existencia de objetivos y planes coherentes y realistas.

- Evaluar la existencia de políticas adecuadas y el cumplimiento de las mismas.

- Comprobar la confiabilidad de la información y de los controles establecidos.

- Verificar la existencia de métodos o procedimientos adecuados de operación y la eficiencia de los mismos.

- Comprobar la utilización adecuada de los recursos. (p.68-69) 
Para llevar a cabo estos objetivos se hace inevitable requerir un marco metodológico que sirva de guía a los pasos en sus diferentes etapas. Según Franklin (2007) estas etapas son:

Planeación: establece el objetivo de la auditoría, los factores a revisar, que incluyen el proceso administrativo y los elementos específicos que complementan su funcionamiento, las fuentes de estudio internas y externas, la investigación preliminar, la preparación del proyecto de auditoría, que considera la propuesta técnica, el programa de trabajo y los aspectos de capacitación, asignación de responsabilidad y actitud, así como el diagnóstico preliminar.

Instrumentación: determina cómo recopilar la información a través de técnicas de recolección como la investigación documental, observación directa, acceso a redes de información, entrevistas, cuestionarios y cédulas, se toman en cuenta los aspectos de medición como escalas e indicadores, los papeles de trabajo del auditor, la evidencia que sustenta los hallazgos y la supervisión del trabajo en sus diferentes modalidades.

Examen: establece el propósito, el procedimiento, los enfoques de análisis administrativo, tanto organizacionales como cuantitativos seleccionados para orientar el contexto de la información captada, y la formulación del diagnóstico administrativo, en el cual se incorporan los aspectos que servirán para evaluar los hechos, las tendencias y situaciones para consolidar un modelo analítico y distintivo de organización. 
Narda P. Merchán-Ponce; Nancy F. Pinargote-Vásquez; Erick R. Baque-Sanchez; Gloria P. Chiquito-Tigua; Ruben D. AceboCalderón; Lady L. Paredes-Acebo

Informe: se registran los resultados de la auditoría, los aspectos operativos acordados para orientar su ejecución, los lineamientos generales para su preparación, los tipos de informe que se pueden utilizar, tales como el general, el ejecutivo y el de aspectos relevantes, y la propuesta de implementación.

Seguimiento: se integran las observaciones y recomendaciones, para lo cual se precisa un objetivo, lineamientos generales y acciones específicas para examinar el grado de avance en la implementación de los cambios sugeridos y sentar las bases para realizar una auditoría de seguimiento. (p. 124-125).

Franklin. (2007) indica la importancia de establecer metodologicamente los parametros para iniciar un proceso de auditoria en una empresa, afirmando que

El marco metodológico representa el camino indicado para guiar las acciones de una auditoría administrativa y la alternativa de brindar a una organización los elementos de juicio idóneos para transformar las experiencias en conocimiento. También es una oportunidad para evaluar estratégicamente el desempeño de manera crítica y objetiva, fortalecer las capacidades distintivas y crear las condiciones para un crecimiento sostenido. (p. 125).

Para este seguimiento, verificación, evaluación y comprobación requerimos utilizar indicadores de gestión que son una herramienta con la que podamos establecer parámetros que nos permitan medir si se va en la dirección correcta de alcanzar los objetivos y metas planteadas. 
Luna \& Morocho. (2012) establecen que "el objetivo de los indicadores de gestión es poder evaluar el desempeño de área mediante parámetros establecidos en relación con las metas, así mismo observar la tendencia en un lapso de tiempo durante el proceso de evaluación" (p. 24).

Los indicadores presentan una serie de características y parámetros. Según Luna \& Morocho. (2012) "las características que debe reunir de un indicador son: relevante, fácil de medir, trasmitir fácilmente la información, verificable, aceptado por la entidad, fácil interpretación, tener precisión matemática y conceptual de los indicadores” (p. 24), los mismos autores definen a los parámetros como los aspectos a evaluar en un enfoque sistémico de gestión de una unidad u organización. Son parámetros de gestión "efecto, impacto, eficacia, eficiencia, economía y calidad” (p. 25).

\section{Materiales y métodos.}

El tipo de estudio es documental y bibliográfico, Documental ya que utiliza como método la recolección de información con el cual se establecen análisis y resultados acordes al objeto a estudio, se recurrió a libros, investigaciones, tesis, medios digitales y/o electrónicos como artículos de opinión y páginas web. Bibliográfico, dado que se procura fortalecer el objeto a estudio y en futuro pueda seguir siendo investigado y desarrollado.

\section{Resultados.}

La auditoría de gestión necesita, al igual que la auditoría financiera, ser lo más sistemática posible en la realización de sus trabajos. La sistematización precisa un método de uso general aplicable a cualquier área, estructura, funciones, etc., que se audite. Este método consiste en un análisis en profundidad y con los máximos detalles utilizando las preguntas clásicas: Qué se quiere, cómo y cuándo se quiere, 
Narda P. Merchán-Ponce; Nancy F. Pinargote-Vásquez; Erick R. Baque-Sanchez; Gloria P. Chiquito-Tigua; Ruben D. Acebo-

dónde, por qué, qué medios se deben utilizar, etc., realizando, además, las siguientes investigaciones: Estudio de los elementos que componen el sector auditado, diagnóstico detallado, determinación de fines y medios, investigación de las deficiencias, balance analítico, comprobación de la eficacia, búsqueda de problemas, proposición de soluciones, indicación de alternativas y mejora de la situación. (Redondo, Llopart, \& Duran, 1996, p. 10).

La auditoría, tradicionalmente hasta el año sesenta, estuvo orientada fundamentalmente al examen de los estados financieros. Con el surgimiento de la Auditoría Operacional como un nuevo campo de ejercicio profesional, se denominó a la Auditoría Tradicional como auditoría de Estados Financieros o Auditoría Financiera, la cual con respecto a la Auditoría Operacional presenta múltiples diferencias relacionadas con el objetivo, el alcance, la orientación, los parámetros o criterios de medición, el método; los interesados, quienes la ejecutan, el contenido del informe, el objetivo de la evaluación del control interno. (Bernal, 2009, p. 2). 


\section{Imagen $N^{\circ}$ 1: Diferencias entre auditoría financiera y operacional}

\begin{tabular}{|c|c|c|}
\hline Con respecto a: & A. Financiera & A. Operacional \\
\hline Objetivo & $\begin{array}{l}\text { Emitir opinión o dictamen sobre la razonabilidad de la situación finan- } \\
\text { ciera de una entidad a una fecha dada, así como sobre el resultado } \\
\text { de sus operaciones financieras y el flujo de fondos para el periodo } \\
\text { que termina. } \\
\text {-Proporcionar un grado de confiabilidad de dichos estados para usarlos } \\
\text { en la toma de decisiones administrativas o gerenciales y en el proceso } \\
\text { de dar cuenta de la gestión. }\end{array}$ & $\begin{array}{l}\text { - Determinar el grado de eficacia, economia y eficiencia de las opera- } \\
\text { ciones para formular las recomendaciones para corregir deficiencias } \\
\text { o para mejorar dicho grado de eficacia, economía y eficiencia. } \\
\text { Contribuir al logro de la prosperidad razonable de la empresa o } \\
\text { entidad. }\end{array}$ \\
\hline Alcance & Limitado a las operaciones financieras. & $\begin{array}{l}\text { - llimitado. Puede cubrir todas las operaciones o actividades que realiza } \\
\text { un organismo, entidad o empresa. }\end{array}$ \\
\hline Orientación & $\begin{array}{l}\text { Hacia la situación financiera y resultados de las operaciones de la entidad } \\
\text { desde un punto de vista retrospectivo. }\end{array}$ & $\begin{array}{l}\text { - Hacia las operaciones o actividades de la entidad en el presente con } \\
\text { proyección el futuro. }\end{array}$ \\
\hline Medición & Principios de contabilidad generalmente aceptados. & - No existen aún criterios de medición de aceptación general. \\
\hline Método & - Las nommas de auditoría generalmente aceptadas. (NACAs). & $\begin{array}{l}\text { - No existen todavía normas de Auditoria Operacional generalmente } \\
\text { aceptadas. El método se detenmina de acuerdo al criterio del audi- } \\
\text { tor operacional. Pueden adaptarse normas de Auditoría Financiera } \\
\text { generalmente aceptadas. }\end{array}$ \\
\hline Interés & $\begin{array}{l}\text { Principalmente extemos: Accionistas, Gobiemo, Bancos; Entidades de } \\
\text { Crédito e Inversiones. }\end{array}$ & $\begin{array}{l}\text { - Desde el punto de vista interno: la alta dirección. Desde el punto de } \\
\text { vista extemo. los accionistas y el gobiemo y la comunidad, cuando } \\
\text { se trata de una entidad del sector público. }\end{array}$ \\
\hline $\begin{array}{l}\text { Quienes la } \\
\text { Ejecutan }\end{array}$ & $\begin{array}{l}\text { - Los contadores públicos con personal de apoyo de la misma pro- } \\
\text { fesión. }\end{array}$ & $\begin{array}{l}\text { - Contadores públicos con participación del personal de la misma } \\
\text { profesión y de profesionales de otras disciplinas. }\end{array}$ \\
\hline $\begin{array}{l}\text { Contenido del } \\
\text { Informe }\end{array}$ & $\begin{array}{l}\text { - Su contenido está exclusivamente relacionado con los estados } \\
\text { financieros. }\end{array}$ & $\begin{array}{l}\text { - Su contenido está relacionado con cualquier aspecto de importancia } \\
\text { de la administración o de las operaciones de la entidad, organismo } \\
\text { o empresa pública. }\end{array}$ \\
\hline $\begin{array}{l}\text { Objetivo de la } \\
\text { Evaluación del } \\
\text { Control Interno }\end{array}$ & $\begin{array}{l}\text { - Determinar la naturaleza y alcance de los procedimientos de Audi- } \\
\text { toría Financiera a emplear. }\end{array}$ & $\begin{array}{l}\text { - Determinar las áreas débiles o críticas más importantes para hacia } \\
\text { ellas orientar todos los esfuerzos de auditoría operacional. }\end{array}$ \\
\hline
\end{tabular}

Fuente: (Bernal, 2009).

Las causas que originan las observaciones de auditoria son aquellas que principalmente se encuentran fuera del alcance de las actividades de control. La causa fundamental de la observación esta encubierta y su ubicación requiere persistencia y conocimiento, si en esta no se identifica la causa de una observación, se pone en peligro la auditoria al desconocer sus implicaciones potenciales en los hallazgos. (Andrade \& Salomé, 2013, p. 123).

\section{Conclusiones.}

- Esta técnica o herramienta permite ver completamente las interacciones de las empresas u organizaciones para el análisis de las operaciones empresariales. 
Narda P. Merchán-Ponce; Nancy F. Pinargote-Vásquez; Erick R. Baque-Sanchez; Gloria P. Chiquito-Tigua; Ruben D. Acebo-

- Realiza una revisión de la estructura organizativa, así como los sistemas de gestión de la empresa.

- Facilita la retroalimentación de la información para la toma de decisiones.

- Optimiza el proceso de control y seguimiento de los planes, objetivos y metas; para realizar cualquier corrección que se amerite.

- Verifica la utilización adecuada de los recursos a través de la eficiencia, eficacia y la economía.

- La auditoría de gestión proporciona una técnica para el establecimiento de estrategias correspondientes con los objetivos empresariales.

\section{Recomendaciones}

De acuerdo a las conclusiones obtenidas se establecen las siguientes recomendaciones:

- Las empresas que requieran mejorar su gestión, pueden aplicar esta técnica de auditoría como estrategia de competitividad en el mercado.

- También se recomienda que se realicen nuevas investigaciones aplicando la mencionada técnica a casos específicos a fin de contrastar la gestión realizada con los objetivos estratégicos de la empresa. 


\section{Bibliografía}

Andrade, V., \& Salomé, D. (2013). Auditoría de gestión a los procesos contables, importaciones y crédito de la empresa sistemas de agua potable Quelle SA ubicada en la provincia de Pichincha, Distrito Metropolitano de Quito al 31 de diciembre de 2012.

Bernal, F. (2009). Auditoría Operacional. Actualidad Empresarial.

Cubero, T. (2009). Manual específico de auditoría de gestión. Cuenca: Unidad de Auditoría Interna, Ilustre Municipalidad de Cuenca.

Franklin, E. (2007). Auditoria administrativa: gestión estratégica del cambio. Pearson Prentice-Hall.

Luna, D., \& Morocho, M. (2012). Auditoría de Gestión para la empresa "Comercial Luna".

Redondo, R., Llopart , X., \& Duran, D. (1996). Auditoría de gestión.

Stoner, J., Freeman, R, E., \& Gilbert, D. (1996). Administración. Pearson Educación. 